Review

\title{
Intensity-Modulated Optical Fiber Sensors Based on Chirped-Fiber Bragg Gratings
}

\author{
Xinyong DONG \\ Institute of Optoelectronic Technology, College of Optical \& Electronic Technology, China Jiliang University, \\ Hangzhou, 310018, China \\ *Corresponding author: Xinyong DONGＥ-mail: xydong@cjlu.edu.cn
}

\begin{abstract}
Intensity-modulated fiber Bragg grating (FBG) sensors, compared with normal wavelength-encoding FBG sensors, can reduce the cost of sensor system significantly by using cost-efficient optical power detection devices, instead of expensive wavelength measurement instruments. Chirped-FBG (CFBG) based intensity-modulated sensors show potential applications in various sensing areas due to their many advantages, including inherent independence of temperature, high measurement speed, and low cost, in addition to the merits of all fiber-optic sensors. This paper theoretically studies the sensing principle of CFBG-based intensity-modulated sensors and briefly reviews their recent progress in measurement of displacement, acceleration, and tilt angle.
\end{abstract}

Keywords: Optical fiber sensors, fiber Bragg gratings, intensity-modulated sensors

\section{Introduction}

Optical fiber Bragg gratings (FBGs) have attracted considerable interests in various fiber-optic sensor implementations for the last decade due to their sensitive responses to strain and temperature [1, 2]. The reliable linear response of Bragg (or resonant) wavelength of FBG to applied axial strain has facilitated various transducer designs that can deal with a lot of measurands such as pressure $[3,4]$, acceleration [5, 6], displacement [7], bending [8], vibration [9], tilt angle [10-13], electrical current [14], underwater acoustic wave $[15,16]$. In addition to the well-known advantages of fiber-optic sensors such as electrically passive operation, immunity to radio-frequency interference (RFI) and electromagnetic interference (EMI), high sensitivity, compact size, light weight, corrosion resistance, and potentially low cost, FBG-based sensor is one of the promising candidates for sensing applications with its own advantages such as inherent self-referencing capability and being easily multiplexed in a serial fashion along a single fiber. Therefore, FBG-based sensors and sensor systems have been widely studied especially in the area of smart materials and structures, where a large number of distributed and embedded sensor elements are needed to make material or structure "smart".

Generally, FBG sensors are based on the measurement of Bragg wavelength shift of FBGs. Therefore, a wavelength interrogator is necessary to get wavelength shift information continuously. A simple and straightforward method to realize this is to use a spectrometer or an optical spectrum analyzer, but neither is attractive in practical applications due to the big size, high cost and low speed. In most sensor systems, wavelength shift of FBG is converted into some easily-measured

Received: 7 November 2010 / Revised version: 25 November 2010 
parameters, such as amplitude, phase, or frequency, by using different wavelength interrogators, which may be based on various techniques such as linearly wavelength-dependent optical filters, scanning Fabry-Perot filters, tunable acousto-optic filters, matched fiber grating pairs, and unbalanced MachZehnder interferometers [17]. These wavelength interrogators, however, increase the complexity and cost of whole sensing system. In addition, FBGs are intrinsically sensitive to both strain and temperature, thus there is always a problem of cross sensitivity between them. To distinguish between both effects, additional temperature compensation schemes or techniques have to be used in the system. This may also increase the cost and complexity of the system.

Since wavelength interrogator and temperature compensator are indispensable in wavelengthencoded FBG sensor systems, it is natural that one looks into a non-wavelength-encoded sensing technique to reduce the cost and to simplify the system. In our previous study [8], the bandwidth of chirped-FBG(CFBG) was used to track the bending state of a flexible beam, on which the FBG was attached and chirped by strain filed produced on the beam lateral side. This method showed the advantage of temperature insensitivity. The bandwidth of chirped-FBG was not changed by temperature because every part of FBG had equal response to temperature variation. However, bandwidth measurement still needs wavelength measurement.

A much better method is reported by using the FBG-reflected optical power as the tracking signal [18-21]. In this method, measurands are related to the bandwidth and thus optical power of the reflected light of the FBG in various ways. The advantage of temperature insensitivity is remained while there is no need for wavelength measurement. This type of sensor will be truly "smart", at least in that an aspect of self-compensation of temperature, eliminating the need for additional instrumentation. However, previous reports presented only their specific sensor designs and results, failed to give a theoretical analysis of the basic sensing principle and the requirements for FBGs. In this paper, the basic working principle is analyzed and the sensor performance is studied in terms of various FBG parameters. Additionally, several sensor designs developed recently based on this method are introduced.

\section{Theoretical study}

The proposed sensor prototype consists of one or more FBGs with initially uniform period, which are chirped by nonuniform strain fields related to the measurand so that their bandwidths, reflectivity and therefore reflected optical powers are changed. By detecting the variation with a photodetector or an optical power meter, measurement of the measurand can be realized.

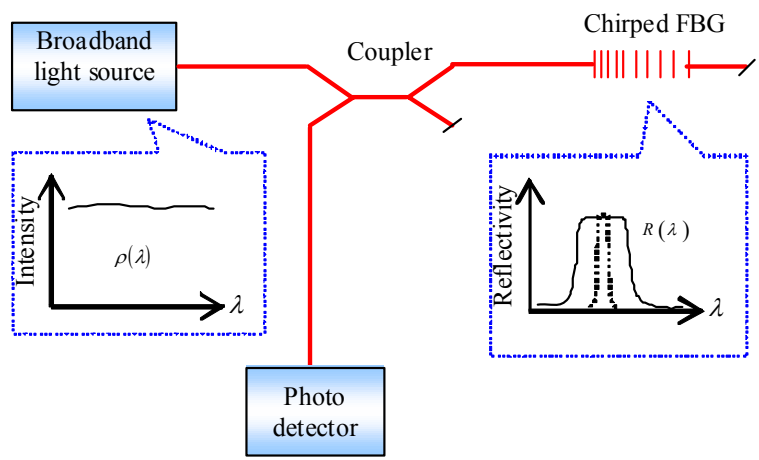

Fig. 1 Interrogation system of the proposed sensor prototype.

Figure 1 shows the schematic diagram of interrogation system of the proposed sensor prototype. Light from a broadband source is injected into the chirp-tuned FBG through an optical coupler. Reflected light from grating is then guided via the same coupler and measured by a photo detector (or power meter). The detected optical power can be expressed as

$$
P=V \int_{\lambda} \rho(\lambda) R(\lambda) d \lambda
$$

where $V$ is a factor that describes the total loss of reflected light from the source to the detector, including the losses arising from fiber splicing and connection, insertion loss of the coupler (the detected light passes the coupler twice before being 
measured), etc, but excluding transmission loss of the grating. $\rho(\lambda)$ and $R(\lambda)$ are the power spectral density of the broadband light source and the reflectivity of grating, respectively. Both are functions of wavelength $\lambda$.

It is notable that the function $R(\lambda)$, which also includes the information of grating, will vary with measurand. $\rho(\lambda)$ is supposed to be a constant, which means that it is not changeable in the sensing process and it does not change with wavelength. Such flat-output, broadband light source can be realized by using a super-luminescent light emitted diode or an output-flattened amplified spontaneous emission source with pumped erbium-doped fiber. And for most cases, the reflection band of the chirped FBGs can be regarded as rectangular shapes. With above assumptions, (1) can be therefore rewritten as

$$
P=V \rho R \Delta \lambda_{\mathrm{bw}}
$$

where $\rho$ is the constant power spectral density of broadband light source, $R$ is the reflectivity and $\Delta \lambda_{\text {bw }}$ is $3-\mathrm{dB}$ bandwidth of grating. Since temperature variation cannot change the reflection profile but the central wavelength of sensing grating, the detected optical power will inherently insensitive to temperature.

Based on coupled mode theory, a uniform FBG usually reflects light within a very narrow wavelength band with Bragg wavelength defined by following expression:

$$
\lambda_{\mathrm{B}}=2 n_{\mathrm{eff}} \Lambda
$$

where $n_{\text {eff }}$ is effective refractive index of fiber core, and $\Lambda$ is grating period (i.e. the period of index perturbation). As it is known, the strain response of FBG arising from both the physical elongation of grating, corresponding to the fractional change in grating period, $\Delta \Lambda$, and the change in fiber index, $\Delta n_{\text {eff }}$, due to photoelastic effects, can be expressed as [1]

$$
\frac{\Delta \lambda_{\mathrm{B}}}{\lambda_{\mathrm{B}}}=\frac{\Delta n_{\mathrm{eff}}}{n_{\mathrm{eff}}}+\frac{\Delta \Lambda}{\Lambda}=\left(1-p_{\mathrm{e}}\right) \varepsilon_{\mathrm{ax}}
$$

where $\Delta \lambda_{\mathrm{B}}$ is the change of Bragg wavelength,
$\varepsilon_{\mathrm{ax}}=\Delta \Lambda / \Lambda$ is the axially applied strain along FBG, and $p_{\mathrm{e}}$ is effective photoelastic constant (about 0.22) of optical fiber material.

It can be seen from above equation that the variation in Bragg wavelength of an FBG is directly proportional to the applied axial strain. If we apply a linearly varying strain field along FBG and assume that this FBG is composed of many small grating segments, the change in Bragg wavelength of each small grating segment will be different, depending on local strain level. That introduces a chirp to grating period with a chirp rate depending on gradient of the applied strain field. The reflection band will be broadened and the bandwidth will be decided by the magnitude of the introduced chirp rate. For the convenience of analysis, here we express the linearly varying strain field as

$$
\varepsilon_{\text {ax }}(z)=\varepsilon_{0}+K_{\varepsilon} z \quad\left(-0.5 L_{\mathrm{g}} \leq z \leq 0.5 L_{\mathrm{g}}\right)
$$

where $z$ is the axis along grating length and $z=0$ corresponds to the center of the grating, $\varepsilon_{0}=\varepsilon_{\mathrm{ax}}(0)$ is the strain applied at the center of fiber grating, $K_{\varepsilon}$ is the gradient of the strain field, and $L_{\mathrm{g}}$ is the length of the fiber grating. By substituting (5) into (4), we can get

$$
\Delta \lambda_{\mathrm{B}}(z)=\Delta \lambda_{\mathrm{B} 0}+\lambda_{\mathrm{B}} K_{\varepsilon}\left(1-p_{\mathrm{e}}\right) z
$$

where $\quad \Delta \lambda_{\mathrm{B} 0}=\Delta \lambda_{\mathrm{B}}(0)=\lambda_{\mathrm{B}}\left(1-p_{\mathrm{e}}\right) \varepsilon_{0} \quad$ is $\quad$ Bragg wavelength shift at the center of grating. The strain-induced variable chirp rate and bandwidth of grating reflection hence can be given respectively by

$$
R_{\mathrm{ch}}=\frac{d\left(\Delta \lambda_{\mathrm{B}}\right)}{d z}=\lambda_{\mathrm{B}} K_{\varepsilon}\left(1-p_{\mathrm{e}}\right)
$$

and

$$
\Delta \lambda_{\mathrm{bw}}=\Delta \lambda_{\mathrm{bw} 0}+R_{\mathrm{ch}} L_{\mathrm{g}}=\Delta \lambda_{\mathrm{bw} 0}+\lambda_{\mathrm{B}} K_{\varepsilon} L_{\mathrm{g}}\left(1-p_{\mathrm{e}}\right)
$$

where $\Delta \lambda_{\text {bw0 }}$ is the initial bandwidth of grating reflection. So the bandwidth of the strain-tuned uniform FBG is changed linearly with the gradient of the applied strain field. But to evaluate the reflected light power from FBG, we also need to know details of the reflectivity, which may be reduced after the grating is chirped.

In the following we numerically study the reflection spectrum of the chirp-rate-tuned FBG by 
using transfer matrix method, and study the relationship between reflected optical power and bandwidth depending on the parameters of the initially uniform FBG. All the parameters including grating length $L_{\mathrm{g}}$, effective refractive index $n_{\text {eff }}$, central Bragg wavelength $\lambda_{\mathrm{B}}$ and index modulation depth $v \overline{\delta n}_{\text {eff }}$ are fixed, but the phase term in the description of "dc" coupling coefficient is varied to achieve different chirp rates [22].

Figure 2 shows several calculated reflection spectra of FBGs with different chirp rates. The narrowest reflection spectrum is for zero chirp rate, corresponding to the originally uniform FBG. In this case, $3-\mathrm{dB}$ bandwidth is $0.5 \mathrm{~nm}$ and the reflectivity is 1 . With the chirp rate increased, the bandwidth is increased along with reduced reflectivity. The reduction in reflectivity is small (less than 0.005) until the bandwidth is broadened to more than $4.2 \mathrm{~nm}$. Details of reflectivity against $3-\mathrm{dB}$ bandwidth are shown in Fig. 3.

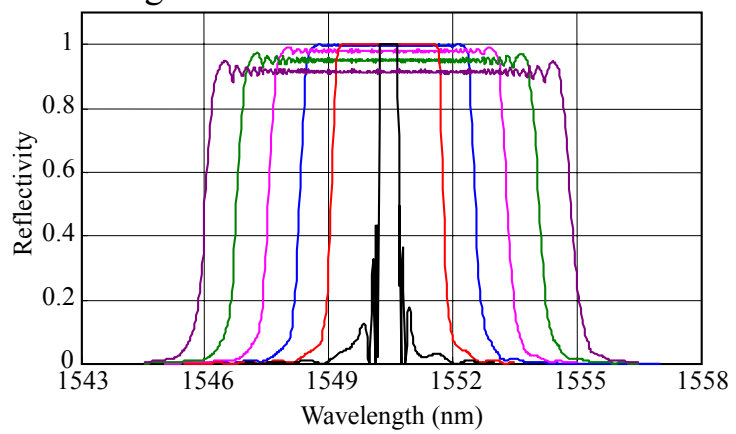

Fig. 2 Simulated reflection spectra of chirp-rate-tuned FBG: $n_{\text {eff }}=1.45, v \overline{\delta n}_{\text {eff }}=4 \times 10^{-4}, L_{\mathrm{g}}=4 \mathrm{~cm}$, and $\lambda_{\mathrm{B}}=1550 \mathrm{~nm}$.

By assuming a constant power spectral density of $\rho=1 \mathrm{~mW} / \mathrm{nm}$ and a loss factor of $V=0.2$, optical powers can also be calculated out by using (2). The calculated optical power data against 3-dB bandwidth are also shown in Fig. 3. A good linear response of $0.19 \mathrm{~mW} / \mathrm{nm}$ can be maintained till a wide bandwidth of $7.3 \mathrm{~nm}$. Over this bandwidth, the response becomes nonlinear due to obvious reduction in reflectivity of chirped FBG.

Further studies have been carried out to study the effects of index modulation depth due to grating inscription, as well as grating length, on the reflectivity and reflected optical power. Figures 4 and 5 show calculated reflectivity against $3-\mathrm{dB}$ bandwidth for chirp-rate-tuned FBG with various $v \overline{\delta n}_{\text {eff }}$ and $L_{\mathrm{g}}$, respectively. Other parameters are the same as those used in aforementioned case.

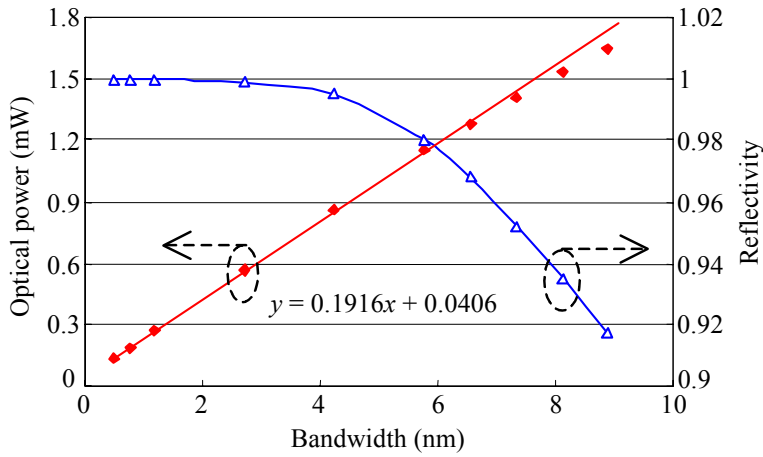

Fig. 3 Calculated reflectivity and detectable optical power against 3-dB bandwidth of chirp-tuned FBG: $n_{\text {eff }}=1.45$, $v \overline{\delta n}_{\text {eff }}=4 \times 10^{-4}, L_{\mathrm{g}}=4 \mathrm{~cm}, \lambda_{\mathrm{B}}=1550 \mathrm{~nm}, \rho=1 \mathrm{~mW} / \mathrm{nm}$, and $V=0.2$.

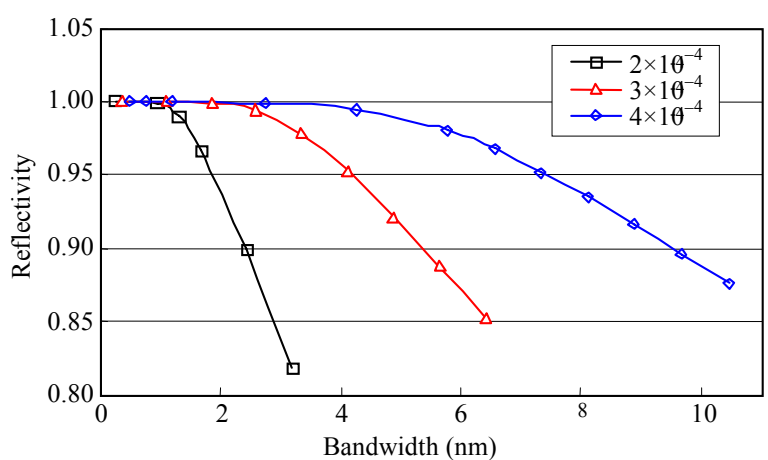

Fig. 4 Reflectivity against bandwidth for chirp-rate-tuned FBG with various $v \overline{\delta n}_{\text {eff }}: n_{\text {eff }}=1.45, L_{\mathrm{g}}=4 \mathrm{~cm}$, and $\lambda_{\mathrm{B}}=1550 \mathrm{~nm}$.

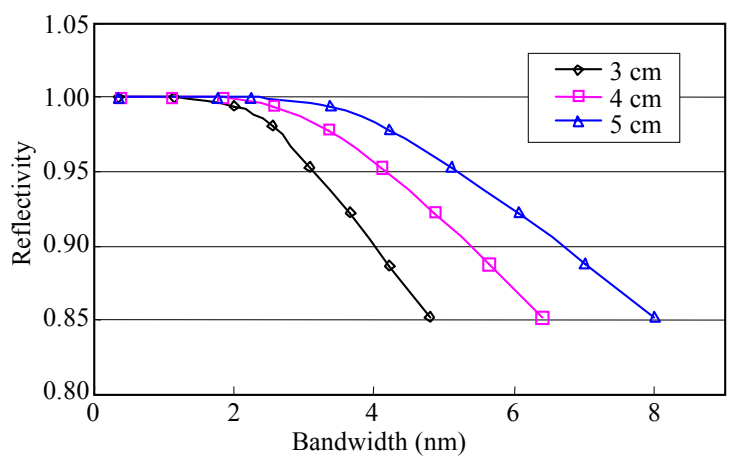

Fig. 5 Reflectivity against bandwidth for chirp-rate-tuned FBG with various grating lengths: $n_{\mathrm{eff}}=1.45, L_{\mathrm{g}}=4 \mathrm{~cm}$, and $\lambda_{\mathrm{B}}$ $=1550 \mathrm{~nm}$

It is obviously shown that a strong FBG with larger index modulation depth and longer length can 
maintain high reflectivity in a larger bandwidth range. Since maintaining higher reflectivity is good for extension of the linear response range of the measured optical power signal, strong and long FBGs are more preferable than weak and short ones in this sensor prototype.

\section{CFBG-based displacement sensor [23]}

To measure displacement using intensitymodulated, CFBG-based technique, the variation of displacement should be related to the bandwidth of FBG sensor. To achieve that, we use a specially designed right-angled triangular cantilever beam, on which the FBG is attached on the lateral side at a slant orientation, so that a varying strain field is generated and transferred to the grating along the length, resulting in a variable but uniform chirp rate in grating period [24].

The schematic diagram of the proposed FBGbased displacement sensor as well as the experimental setup is shown in Fig. 6. An originally uniform 10-cm-long FBG is fabricated by exposing a deeply hydrogen-loaded single-mode fiber to a 244-nm laser beam through a phase mask. The fabricated grating has a very high reflectivity of about $100 \%$. The original $3-\mathrm{dB}$ bandwidth and the center wavelength of the grating are $0.26 \mathrm{~nm}$ and $1550.9 \mathrm{~nm}$, respectively. The right-angled triangular cantilever beam has length $L_{b}=18 \mathrm{~cm}$, thickness $h=$ $0.8 \mathrm{~cm}$, and width of the fixed end $b_{0}=3 \mathrm{~cm}$. The angle between the axis of the grating and the neutral layer of the beam is $\theta=4.5^{\circ}$.

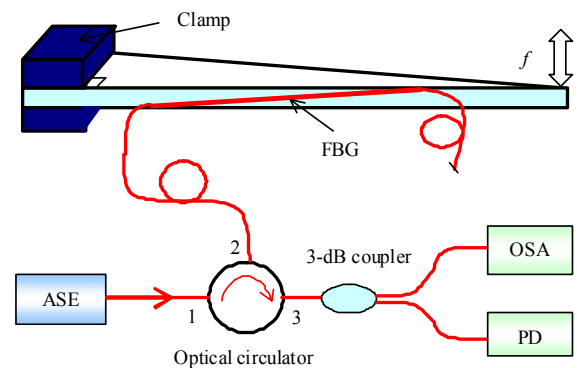

Fig. 6 Schematic diagram and experimental setup of the proposed chirp-rate-tuned FBG based displacement sensor: ASE, gain-flattened amplified spontaneous emission source; OSA, optical spectrum analyzer; PD, photodetector.
When the cantilever beam is bent by applying a vertical displacement (or a force) on the free end, half of the grating is under a varying tension whereas the other half is under a varying compression. The strain on the neutral layer of the beam is zero. If the center of grating is located well to the neutral layer of the beam, there will be no strain effect on the center of grating. Therefore, the center wavelength of chirped grating may keep fixed since the strain applied to two halves of the grating is symmetrical.

An output power flattened amplified spontaneous emission (ASE) source was used as input light to fiber grating via Port 1 of an optical circulator, which directed light to Port 2 and then to the fiber grating. The reflected light from Port 3 of the circulator was measured with both optical spectrum analyzer (OSA) and photodetector (PD) through a 3-dB fiber coupler.

The relationship between reflection bandwidth of FBG and the applied vertical displacement $f$ at the free end of the cantilever beam can be given by

$$
\Delta \lambda_{\mathrm{bw}}=\Delta \lambda_{\mathrm{bw} 0}+\frac{\mathrm{C} \lambda_{\mathrm{B}} L_{\mathrm{g}}}{L_{b}^{2}} \sin (2 \theta)\left(1-p_{\mathrm{e}}\right) f
$$

where $\mathrm{C}(0<\mathrm{C}<1)$ is a constant that represents the efficiency of strain transfer from the beam to FBG.

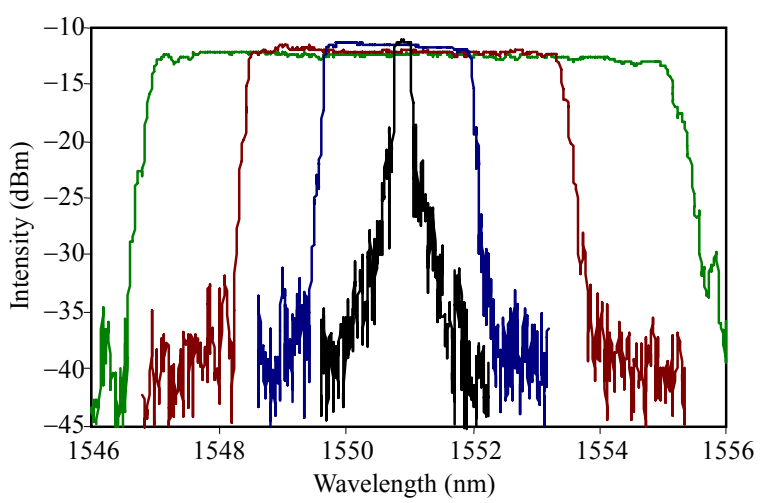

Fig. 7 Reflection spectra of CFBG sensor under different displacements.

Figure 7 shows the measured spectra of the chirped FBG for different displacements of $0,6 \mathrm{~mm}$, $13 \mathrm{~mm}$, and $22 \mathrm{~mm}$. The measured 3-dB bandwidth and PD output signal for displacements varying 
from 0 to $15 \mathrm{~mm}$ are shown in Fig. 8. A linear fit to the data of $3-\mathrm{dB}$ bandwidth gives a high $R$-squared value of 0.9999 , showing a good linearity of the response of bandwidth with displacement. For PD output signal, linear response over 0.999 is achieved within the displacement range of $9 \mathrm{~mm}$. The response of PD output to displacement is $37.9 \mathrm{mV} / \mathrm{mm}$.

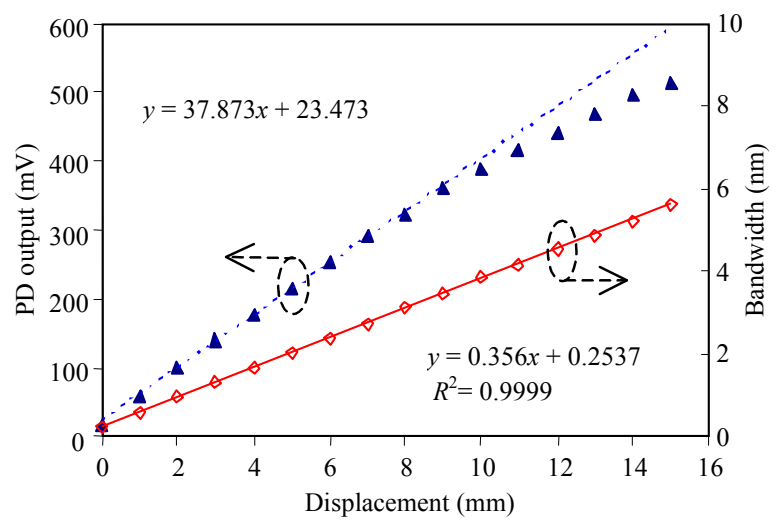

Fig. 8 PD output and 3-dB bandwidth against displacement.

To test the temperature stability of the sensor, we measured FBG spectrum and PD output for a constant displacement of $5 \mathrm{~mm}$ at various temperatures. The center wavelength of FBG and PD output as a function of temperature are shown in Fig. 9. When temperature increased from $-15{ }^{\circ} \mathrm{C}$ to $40{ }^{\circ} \mathrm{C}$, the center wavelength of FBG shifted to longer wavelength region by $5.3 \mathrm{~nm}$ due to thermal effect. The variation in 3-dB bandwidth was very small (less than $0.1 \mathrm{~nm}$ ). The largest fluctuation of PD output signal of about $220 \mathrm{mV}$ was less than $20 \mathrm{mV}$.

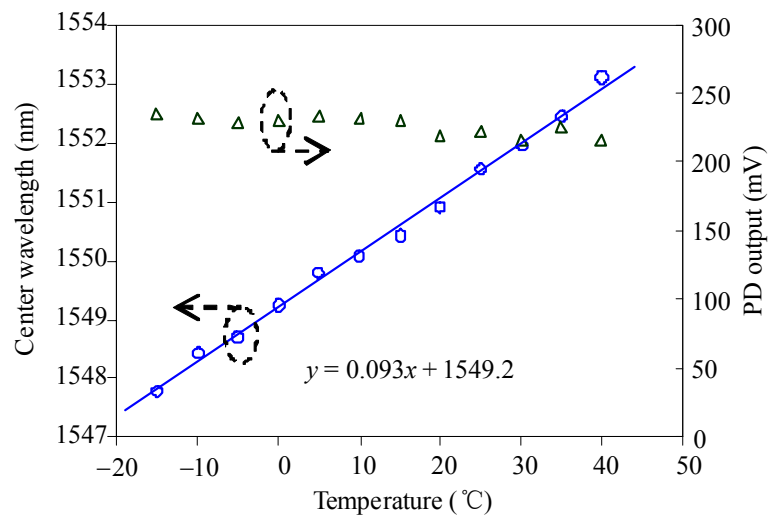

Fig. 9 PD output and center wavelength against temperature under a fixed displacement of $5 \mathrm{~mm}$.

\section{CFBG-based acceleration (vibration) sensor [25-27]}

With the installation of a weight mass on the free end of the cantilever beam of the above-introduced displacement sensor, the same sensor system can be used to measure acceleration in vertical direction. Figure 10 shows the schematic diagram and experimental system.

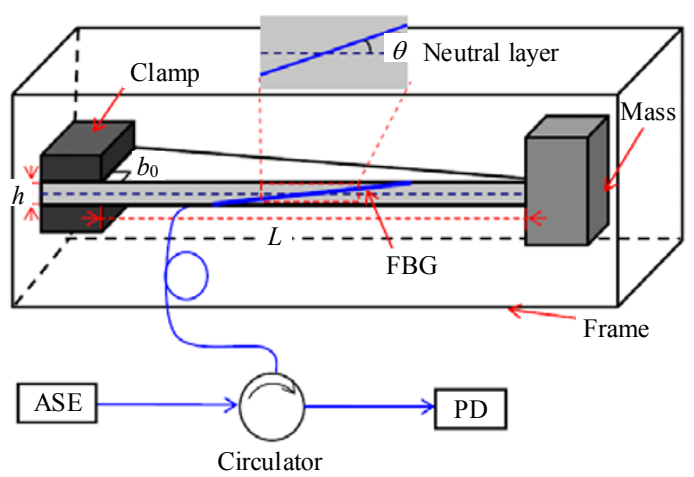

Fig. 10 CFBG-based acceleration (vibration) sensor.

Here we used a 3-cm-length FBG with a high reflectivity over $30 \mathrm{~dB}$, a central Bragg wavelength of $1556.1 \mathrm{~nm}$, and a $3-\mathrm{dB}$ spectral bandwidth of $0.15 \mathrm{~nm}$. The cantilever beam had a length of $16.5 \mathrm{~cm}$, width at the fixed end of $3 \mathrm{~cm}$, thickness of $0.5 \mathrm{~cm}$, and Young's modulus of material of $3.3 \times 10^{9} \mathrm{~Pa}$. The mass's weight was $100 \mathrm{~g}$. The angle $\theta$ was $9.6^{\circ}$, which was changeable depending on the length of FBG and the thickness of the cantilever beam. The cantilever beam was fixed in a frame for the convenience of accelerometer measurement.

The measured 3-dB bandwidth and reflected optical power versus applied vertical acceleration on sensor setup are shown in Fig. 11. The achieved sensitivity is $0.68 \mathrm{~nm} / \mathrm{g}$. It is obvious that the variation in reflected power is not linear when the acceleration exceeds $3 \mathrm{~g}$ because of the obvious reduction in the reflectivity of FBG. A FBG with a longer length and a higher reflectivity will be helpful to increase linear response range.

The weight of the mass is also critical to the sensitivity of the accelerometer. Using a heavier mass leads to a bigger sensitivity, but the 
measurement range of acceleration will be reduced because there is a tradeoff between two parameters.

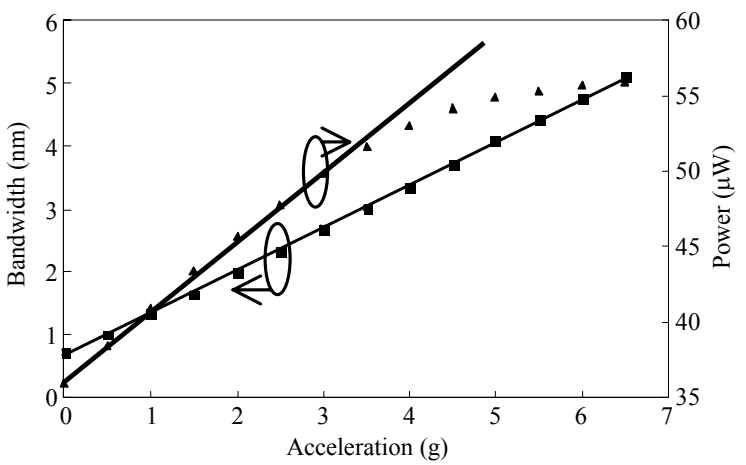

Fig. 11 Measured bandwidth and optical power versus applied acceleration.
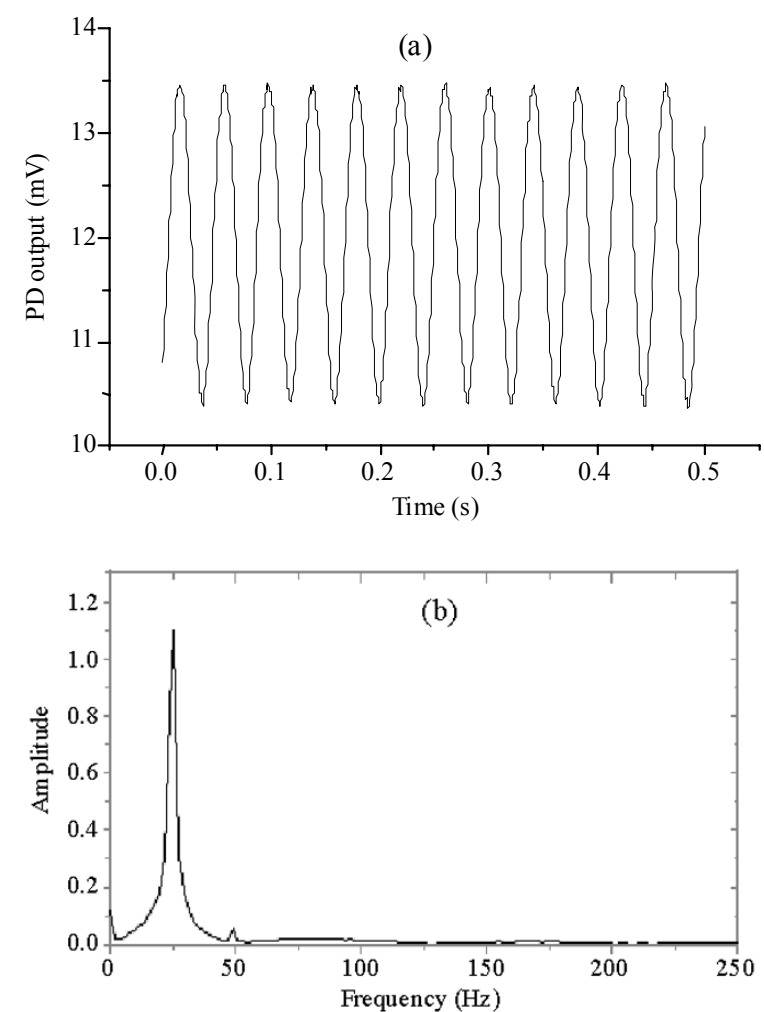

Fig. 12 Measurement results of the vibration sensor: (a) time domain trace and (b) frequency spectrum.

Vibration measurement is carried out by installing an eccentric gear on the free end of the beam as a vibration source. Because the FBG used for experiment was a uniform period grating, the reflection bandwidth would increase whether the deflection was positive or negative. To achieve linear response of FBG, a mass with weight of $200 \mathrm{~g}$ was used to produce an original negative deflection of about $5 \mathrm{~mm}$. Figure 12 shows the measured time domain trace and frequency spectrum of sensor output. Owning to the temperature-independence nature of the reflected optical power of FBG, the sensors are insensitive to temperature.

\section{CFBG-based 2D tilt sensor [28]}

The schematic diagram of the CFBG-based tilt sensor is illustrated in Fig. 13. It consists of two original uniform FBGs attached to the upper tapered part of a cylindrical beam, whose top end is fixed to the sensor frame while the bottom end is bonded with a spherical mass. Two FBGs are glued on the surface of the tapered part of the beam at the same height along the generatrix direction and separated by one fourth of the circumference of the beam from each other. Due to nonuniform tilt-induced strain field applied along the length of FBGs, the bandwidths of their reflection spectra vary linearly with the applied inclination. By monitoring the reflected optical powers of two FBGs, temperature-independent measurement of $2 \mathrm{D}$ tilt angle is realized.

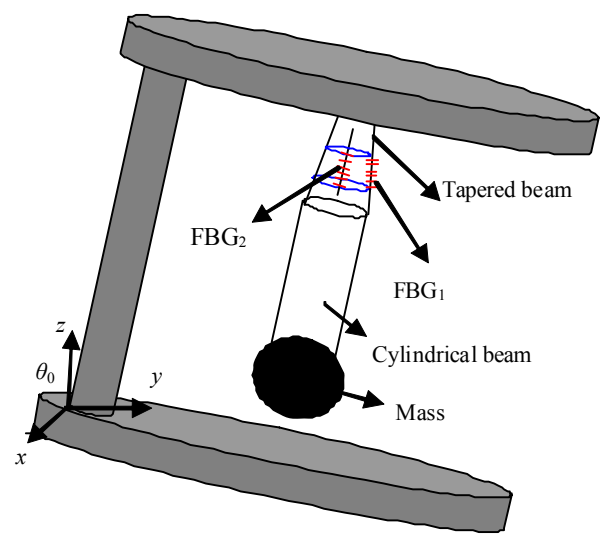

Fig. 13 Schematic diagram of CFBG-based tilt sensor.

For any applied inclination, it can be described by two orthogonal tilt angles. Here, we define that $\theta$ is the angle in $y-z$ plane where $\mathrm{FBG}_{1}$ is located while $\beta$ is the angle in $x-z$ plane where $\mathrm{FBG}_{2}$ is located. When the tilt sensor is inclined at an angle, the beam is bent and nonuniform strain field is produced on the surface of the tapered part of the beam along generatrix direction. Two FBGs are therefore 
chirped and their bandwidths and reflected optical powers are changed.

In order to distinguish the tilt direction, an initial tilt angle, described by $\theta_{0}$ in $y-z$ plane and $\beta_{0}$ in $x-z$ plane, is applied. The sensor setup is then placed at an inclination calibrated platform. Tilt angle $\beta$ is changed first from $-4^{\circ}$ to $4^{\circ}$, while keeping angle $\theta$ at $0^{\circ}$. Figure 14 shows the reflective spectra of $\mathrm{FBG}_{2}$ at three different tilt angles. The measured bandwidths and reflected optical powers of two FBGs against $\beta$ are shown in Fig. 15. The achieved tilt angle sensitivity is $97.1 \mathrm{pm} /{ }^{\circ}$ and $1.96 \mu \mathrm{W} /{ }^{\circ}$. During the measurement, the bandwidth and reflected optical power of $\mathrm{FBG}_{1}$ are nearly unchanged as expected. Similar results are obtained when the experiment is repeated by changing the tilt angle $\theta$ while keeping $\beta$ at $0^{\circ}$.

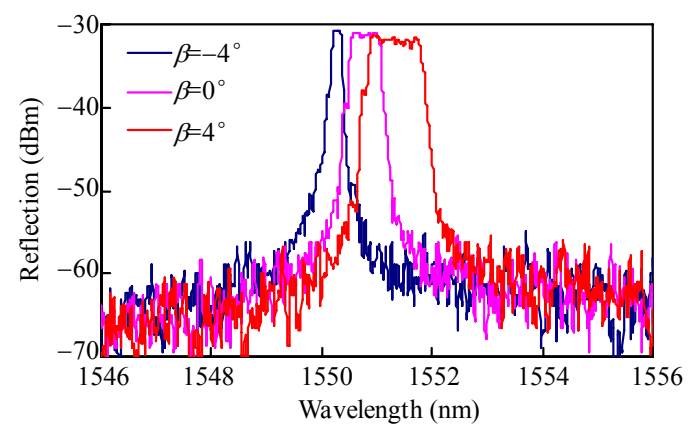

Fig. 14 Reflective spectra of $\mathrm{FBG}_{1}$ at three tilt angles.

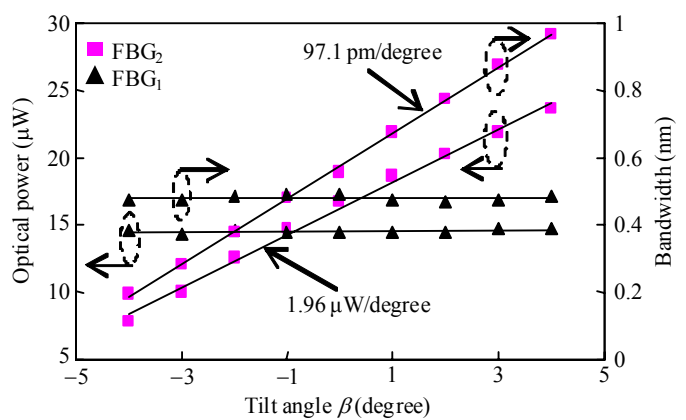

Fig. 15 Optical power and 3-dB bandwidth of two FBGs versus $\beta$ in $x-z$ plane when $\beta$ increased from $-4^{\circ}$ to $4^{\circ}$.

The accuracy of tilt angle measurement is $\pm 0.12^{\circ}$ which is defined as the maximum difference between the applied angle values and calculated values from experimental data. The tilt angle measurement resolution, based on the power measurement resolution of optical power meter and the aforementioned sensitivity, is about $0.027^{\circ}$. Only small fluctuations $\pm 0.15 \mu \mathrm{W}$ (about $0.76 \%$ ) in optical powers are observed in thermal stability measurement in the range of 10 to $50{ }^{\circ} \mathrm{C}$. Therefore, it can be regarded as temperature insensitivity.

\section{Conclusions}

We have theoretically studied the basic sensing principle of an intensity-modulated sensor prototype based on chirp-rate-variable FBGs with the optical power-encoding technique. Long-length and strongreflectivity FBGs are found to be more favorable in this application than other short and weak ones. Several recently developed novel sensor designs for displacement, acceleration (or vibration), and tilt measurement have been presented. These sensors show great advantages including simple construction (no need of wavelength interrogator), low cost, high speed, and inherently insensitive to temperature thus without the need for temperature compensation. These advantages make them useful in a wide range of applications in optical fiber sensor areas.

\section{Acknowledgment}

This work was co-supported by the National Basic Research Program of China (973 Program) under Grant No. 2010CB327804, the National Natural Science Foundation of China under Grant No. 60807021, and the Natural Science Foundation of Zhejiang Province, China under Grant No. R1080087. The author thanks all the collaborators and students who contributed to this work.

Open Access This article is distributed under the terms of the Creative Commons Attribution License which permits any use, distribution, and reproduction in any medium, provided the original author(s) and source are credited.

\section{References}

[1] C. R. Giles, "Lightwave applications of fiber Bragg 
gratings," J. Lightwave Technol., vol. 15, no. 8, pp. 1391-1404, 1997.

[2] Y. J. Rao, "In-fiber Bragg grating sensors," Meas. Sci. \& Technol., vol. 8, no. 4, pp. 355-375, 1997.

[3] M. G. Xu, L. Reekie, Y. T. Chow, and J. P. Dakin, "Optical in-fiber grating high pressure sensor," Electron. Lett., vol. 29, no. 4, pp. 398-399, 1993.

[4] Y. Liu, Z. Guo, Y. Zhang, K. S. Chiang, and X. Dong, "Simultaneous pressure and temperature measurement with polymer-coated fiber Bragg grating," Electron. Lett., vol. 36, no. 6, pp. 564-566, 2000.

[5] T. A. Berkoff and A. D. Kersey, "Experimental demonstration of a fiber Bragg grating accelerometer," IEEE Photon. Technol. Lett., vol. 8, no. 12, pp. 1677-1679, 1996.

[6] M. D. Todd, G. A. Johnson, B. A. Althouse, and S. T. Vohra, "Flexural beam-based fiber Bragg grating accelerometers," IEEE Photon. Technol. Lett., vol. 10, no. 11, pp. 1605-1607, 1998.

[7] X. Dong, Y. Liu, Z. Liu, and X. Dong, "Simultaneous displacement and temperature measurement with cantilever-based fiber Bragg grating sensor," Opt. Commun., vol. 192, no. 3-6, pp. 213-217, 2001.

[8] X. Dong, H. Meng, G. Kai, Z. Liu, and X. Dong, "Bend measurement with chirp of fiber Bragg grating," Smart Mater. Struct., vol. 10, no. 5, pp. 1111-1113, 2001.

[9] K. O. Lee, K. S. Chiang, and Z. H. Chen, "Temperature-insensitive fiber-Bragg-grating-based vibration sensor," Opt. Eng., vol. 40, no. 11, pp. 2582-2585, 2001.

[10] B. O. Guan, H. Y. Tam, and S. Y. Liu, "Temperatureindependent fiber Bragg grating tilt sensor," IEEE Photon. Technol. Lett., vol. 16, no. 1, pp. 224-226, 2004.

[11] H. Bao, X. Dong, C. Zhao, C. C. Chan, and P. Shum, "Temperature-insensitive FBG tilt sensor with a large measurement range," Opt. Commun., vol. 283, no. 6, pp. 968-970, 2010.

[12] S. He, X. Dong, K. Ni, C. C. Chan, and P. Shum, "Temperature-insensitive 2D tilt sensor with three fiber Bragg gratings," Meas. Sci. Technol., vol. 21, no. 2, pp. 025203-025206, 2010.

[13] H. Bao, X. Dong, L. Shao, C. Zhao, and S. Jin, "Temperature-insensitive 2-D tilt sensor by incorporating fiber Bragg gratings with a hybrid pendulum," Opt. Commun., vol. 283, no. 24, pp. 5021-5024, 2010

[14] S. Yang, H. Meng, X. Dong, S. Yuan, and X. Dong, "Electric current measurement with high resolution using FBG covered by aluminum thin film," Proc. SPIE, vol. 4595, pp. 200-203, 2001.

[15] N. E. Fisher, D. J. Webb, C. N. Pannell, D. A. Jackson, L. R. Gavrilov, J. W. Hand, L. Zhang, and I. Bennion, "Ultrasonic field and temperature sensor based on short in-fiber Bragg gratings," Electron. Lett., vol. 34, no.11, pp. 1139-1140, 1998.

[16] L. Y. Shao, S. T. Lau, X. Dong, A. P. Zhang, H. L. W.
Chan, H. Y. Tam, and S. He, "Highly sensitive ultrasonic hydrophone based on a cladding-etched fiber laser," IEEE Photon. Technol. Lett., vol. 20, no. 8, pp. 548-550, 2008.

[17] B. Lee and Y. Jeong, "Interrogation techniques for fiber grating sensors and the theory of fiber gratings," in Fiber optical sensors. F. T. S. Yu and S. Yin Ed., New York: Marcel Dekker, 2002, pp. 295-381.

[18] M. G. Xu, L. Dong, L. Reekie, J. A. Tucknott, and J. L. Cruz, "Temperature-independent strain sensor using a chirped Bragg grating in a tapered optical fiber," Electron. Lett., vol. 31, no. 10, pp. 823-825, 1995.

[19] Y. Zhu, P. Shum, C. Lu, B. M. Lacquet, P. M. Swart, and S. J. Spammer, "Fiber Bragg grating accelerometer with temperature insensitivity," Microwave Opt. Technol. Lett., vol. 37, no. 2, pp. 151-153, 2003.

[20] X. Yang, X. Dong, C. L. Zhao, J.H. Ng, Q. Peng, X. Zhou, and C. Lu, "Temperature-independent measurement of displacement based on the chirp tuning of a fiber grating," Opt. Eng., vol. 44, no. 7, pp. 074401, 2005.

[21] X. Dong, C. Zhan, K. Hu, P. Shum, and C. C. Chan, "Temperature-insensitive tilt sensor with strainchirped fiber Bragg gratings," IEEE Photon. Technol. Lett., vol. 17, no. 11, pp. 2394-2396, 2005.

[22] R. Kashyap, Fiber Bragg gratings. New York: Academic Press, 1999.

[23] X. Dong, X. Yang, C. L. Zhao, L. Ding, P. Shum, and N. Q. Ngo, "A novel temperature- insensitive fiber Bragg grating sensor for displacement measurement," Smart Mater. Struct., vol. 14, no. 2, pp. N7-N10, 2005.

[24] X. Y. Dong, P. Shum, N. Q. Ngo, C. C. Chan, J. H. $\mathrm{Ng}$, and C. L. Zhao, "Largely tunable CFBG-based dispersion compensator with fixed center wavelength," Opt. Express, vol. 11, no. 22, pp. 2970-2974, 2003.

[25] W. Zhou, X. Dong, K. Ni, C. C. Chan, and P. Shum, "Temperature insensitive accelerometer based on a strain-chirped FBG, " Sens., Actuat. A, vol. 257, no. 1, pp. 15-18, 2010.

[26] W. Zhou, X. Dong, C. Shen, C. Zhao, C. C. Chan, and P. Shum, "Temperature-independent vibration sensor with a fiber Bragg grating," Microwave Opt. Technol. Lett., vol. 52, no. 10, pp. 2282-2285, 2010.

[27] L. Li, X. Dong, L. Shao, C. Zhao, and Y. Sun, "Temperature-independent acceleration measurement with a strain-chirped fiber Bragg grating," J. Optoelectron. Adv. Mater., vol. 4, no. 7, pp. 943-946, 2010.

[28] H. Bao, X. Dong, L. Y. Shao, C. Zhao, C. C. Chan, and P. Shum, "Temperature-insensitive pendulum clinometer using two fiber Bragg gratings for 2D tilt angle measurement," IEEE Photon. Technol. Lett., vol. 22, no. 12, pp. 863-865, 2010. 